\title{
Las organizaciones del Tercer Sector Social de Euskadi ante la crisis de la covid-19
}

\section{Lucía Merino}

Observatorio Vasco del Tercer Sector Social

\section{Zuriñe Romeo}

Observatorio Vasco del Tercer Sector Social coordinacion@3seuskadi.org

Euskadiko Hirugarren Sektore Sozialeko (EHSS) erakundeak, berariaz gizarte-kaltebera diren hainbat kolektibo edo komunitateren gizarte-inklusioa eta eskubideak sustatzearen jarduera eraginkorra ezaugarri izaten dutenak, covid-19aren krisialdiaren kudeaketarako alarma-egoeraren hasierako asteetan betetzen duten zereginaren balioa nabarmendu zen. Osasun- eta gizarte-larrialdiko testuinguruak betebetean eragin die bai erakundeen funtzionamenduari bai jardueren xede dituzten zerbitzu-jasotzaileei. Artikulu honetan, lehen aste horietako inpaktuaren ondorioak azaltzen saioa egiten da, alarma-egoeran bai jasotzaile diren pertsonek bai erakundeek aurre eman beharreko problematikak aintzat hartuta (zerbitzuetan, erakundeetako lagunen jardueran eta baliabide-mailan); eta covid-19aren krisialdiak etorkizunean Euskadiko Hirugarren Sektore Sozialean izan dezakeen eragina aztertzeko aukera eskaintzen du.

\section{GAKO-HITZAK:}

Hirugarren sektore soziala, covid-19, efektuak, gizarte-zerbitzuak, jasotzaileak, Euskadi.
Las organizaciones del Tercer Sector Social de Euskadi (TSSE), que se caracterizan por promover la inclusión social y el ejercicio efectivo de los derechos de múltiples colectivos o comunidades en especial vulnerabilidad, demostraron durante las primeras semanas del estado de alarma declarado para la gestión de la crisis de la covid-19 el valor del trabajo que realizan. Este contexto de emergencia sanitaria y social ha incidido de lleno en el funcionamiento de las organizaciones y en las personas destinatarias de su actividad. Este artículo trata de conocer cómo ha sido ese impacto, entendiendo las problemáticas que tanto las personas destinatarias como las organizaciones (sus servicios, sus personas y sus recursos) han tenido que afrontar durante las primeras semanas del estado de alarma, permite entender el calado que tuvo entonces y puede tener a futuro el impacto de la crisis de la covid-19 en el Tercer Sector Social de Euskadi.

\section{Palabras Clave:}

Tercer sector social, covid-19, efectos, servicios sociales, personas destinatarias, Euskadi. 


\section{Introducción}

Este texto se basa en una investigación desarrollada en abril de 2020 por el Observatorio Vasco del Tercer Sector Social ${ }^{1}$ para conocer el impacto de la crisis de la covid-19 en las organizaciones del Tercer Sector Social de Euskadi (en lo sucesivo, TSSE) y, en especial, en sus personas destinatarias, respondiendo a una iniciativa de la Mesa de Diálogo Civil de Euskadi, el Departamento de Empleo y Políticas Sociales del Gobierno Vasco, y Sareen Sarea ${ }^{2}$.

El 14 se marzo de 2020 se declaró el estado de alarma en todo el estado para la gestión de la situación de crisis sanitaria provocada por el Covid-19. Las organizaciones del Tercer Sector Social de Euskadi, que se caracterizan por promover la inclusión social y el ejercicio efectivo de los derechos de múltiples colectivos o comunidades en especial vulnerabilidad, demostraron entonces y siguen demostrando ahora el valor del trabajo que realizan. Ante un contexto de emergencia social de tal envergadura han sido capaces de articularse y coordinarse, de manera urgente y junto a otras instituciones, para paliar los impactos sobre aquellas personas que más han podido verse afectadas por el cambio de escenario que ha supuesto la crisis de la covid-19 (personas mayores, personas en exclusión, infancia vulnerable, mujeres que sufren violencia, etc.) ${ }^{3}$.

Este contexto de emergencia ha incidido de lleno en el funcionamiento de las organizaciones y en las personas destinatarias de su actividad. Por eso, conocer cómo ha sido ese impacto, entendiendo las problemáticas que tanto las personas destinatarias como las organizaciones (sus servicios, sus personas y sus recursos) han tenido que afrontar durante las primeras semanas del estado de alarma, permite entender el calado que tuvo entonces y puede tener a futuro el impacto de la crisis de la covid-19 en el TSSE.

\section{Planteamiento de la investigación}

En la investigación se parte de la definición del Tercer Sector Social que la Ley del Tercer Sector Social de Euskadi recoge y que entiende que forman parte

${ }^{1}$ El Observatorio Vasco del Tercer Sector Social constituye un instrumento técnico de la administración general de la Comunidad Autónoma del País Vasco encargado de la recopilación, sistematización, actualización y generación de información y conocimiento, así como de su difusión, en las esferas relacionadas con el tercer sector social y las organizaciones y redes integradas en él. En el desarrollo de sus atribuciones, el observatorio colaborará estrechamente con las organizaciones y redes.

${ }^{2}$ El informe completo está disponible en la web del Observatorio Vasco del Tercer Sector Social («https://3seuskadi.eus/wp-content/ uploads/Informe_COVID_19.pdf $)$.

${ }^{3}$ Desde el Observatorio Vasco del Tercer Sector Social queremos trasladar nuestro reconocimiento a todas las organizaciones del sector por la labor que han realizado, y siguen haciendo, atendiendo a múltiples realidades desde escenarios complejos y cambiantes. Y nuestro agradecimiento a las entidades que contestaron al cuestionario y a las que participaron en las sesiones online. de él las organizaciones de iniciativa social, con sede y actividad en la Comunidad Autónoma del País Vasco, cuya finalidad principal es promover, a través de actividades de intervención social, la inclusión social, la cooperación al desarrollo y el ejercicio efectivo de los derechos de personas, familias, grupos, colectivos o comunidades que afrontan situaciones de vulnerabilidad o exclusión, desigualdad, desprotección, discapacidad o dependencia. Además, entiende por redes del Tercer Sector Social de Euskadi las organizaciones, de segundo nivel y superiores, representativas de otras, así como las organizaciones de intervención directa del Tercer Sector Social de Euskadi que sean representativas de un determinado colectivo, cuando no exista una red que lo represente en el territorio o ámbito geográfico de actuación de que se trate.

Los objetivos y focos de análisis de la investigación se definieron junto al Departamento de Empleo y Políticas Sociales del Gobierno Vasco y la comisión de la Mesa de Diálogo Civil constituida para responder en colaboración al impacto de la covid-19 en las personas y familias en situación de riesgo, exclusión o pobreza.

La investigación se realizó durante el mes de abril de 2020 y buscaba ofrecer una instantánea del impacto de la crisis en el momento preciso de la recogida de información, un momento de urgencia acuciante en el que las entidades estaban volcadas de lleno en dar respuesta a las múltiples necesidades que se estaban generando.

\section{En concreto, los objetivos del estudio eran:}

1. Medir el impacto de la crisis de la covid-19 en las organizaciones del TSSE, estudiando la afección que estaba teniendo en ese momento (abril 2020) la crisis de la covid-19 en las organizaciones, pero también de la estimación que hacían las entidades con respecto a cómo preveían el impacto a corto plazo (a lo largo del 2020).

2. Analizar el impacto de la crisis de la covid-19 en dos dimensiones concretas:

- En las personas destinatarias de las organizaciones del TSSE, analizando cómo estaba afectando a los proyectos de vida de las personas destinatarias, en qué situación se encontraban, qué problemas estaban afrontando (alojamiento, alimentación, confinamiento, etc.), qué consecuencias pueden acarrear esos problemas en sus vidas, etc.

- En las organizaciones del TSSE, tanto en sus servicios y actividades (cese o cierre de servicios, cancelación de actividades, modificaciones o creación de nuevos servicios o actividades, etc.), como en las personas voluntarias y remuneradas (problemas de seguridad o sanitarios, cambios en las condiciones laborales, etc.) y en sus recursos económicos (disminución de la financiación, problemas de liquidez, etc.). 
3. Conocer la incidencia específica de la crisis de la covid-19 en las organizaciones según su ámbito de actuación o contingencia atendida ${ }^{4}$, tamaño de las organizaciones o territorio histórico en el que se localizan, así como en algunos colectivos concretos de personas destinatarias.

La investigación se sustenta sobre una metodología mixta que combina la recogida de información cuantitativa y cualitativa, a través de diferentes herramientas: cuestionario, grupos de discusión y entrevistas.

\section{El cuestionario tuvo un formato online y estuvo} abierto entre el 9 y el 17 de abril de $2020^{5}$. En el proceso de trabajo se procuró acceder a un número amplio de organizaciones que dieran cuenta de cómo estaban viviendo esta crisis, a la vez que se priorizaba contar con una respuesta rápida para tener información de manera urgente. Dada la premura, se sacrificaron algunos criterios de representatividad de la muestra pero se consiguió llegar a casi 200 organizaciones sociales, de distintos territorios, con actividades diversas, colectivos de personas destinatarias diferentes o distintas según su tamaño, que son muestra de la realidad diversa del TSSE. En total han participado respondiendo al cuestionario 184 organizaciones vascas.

Este estudio debe entenderse como un trabajo de carácter exploratorio, enmarcado en un contexto de urgencia en el que se priorizó ofrecer resultados de manera rápida y ágil. Es importante poner en valor el volumen significativo de respuestas recopiladas en apenas poco más de una semana, que permite realizar un primer acercamiento a la realidad como punto de partida para profundizar posteriormente en el análisis cualitativo. Pese a no atender estrictamente a criterios de representatividad ${ }^{6}$, la

${ }^{4}$ La contingencia diferencia entre organizaciones que atienden a: - Personas en situación o riesgo de dependencia: organizaciones que atienden a personas mayores en situación de dependencia.

- Personas en situación o riesgo de exclusión: organizaciones que atienden a personas en situación o riesgo de pobreza, que ejercen la prostitución, reclusas y exreclusas, con problemas de adicción o drogodependencias, etc., que están vinculadas a la actividad de los servicios sociales.

- Personas en situación o riesgo de desprotección: organizaciones que atienden a infancia en situación de desprotección, mujeres víctimas de maltrato, abusos, etc.

- Personas con discapacidad: organizaciones que atienden a personas con discapacidad, incluye enfermedad mental y algunas enfermedades crónicas, degenerativas, etc.

- Otros colectivos vulnerables: organizaciones de personas mayores, gitanas, inmigrantes, familias, mujeres asociadas, personas con adicciones, infancia y juventud en general, personas con enfermedades (que no conllevan específicamente dependencia o discapacidad) asociadas, etc.

${ }^{5}$ La invitación a participar en el estudio cumplimentando el cuestionario fue lanzada tanto desde el Gobierno Vasco-Observatorio Vasco del Tercer Sector Social como desde Sareen Sarea, animando a sus redes y organizaciones a participar.

${ }^{6}$ Para determinar la fiabilidad y representatividad de la muestra obtenida es preciso tener en cuenta la definición precisa del TSSE ya mencionada y el volumen del universo de organizaciones que lo componen. De acuerdo con los últimos datos publicados sobre el TSSE, se respuesta conjunta de esas 184 organizaciones resulta clave para conocer el impacto que están viviendo tanto las personas destinatarias como las propias entidades?.

Con el fin de completar el análisis cuantitativo de datos, interpretarlos e intercambiar distintas situaciones y experiencias ante la crisis de la covid19, se recurrió a una consulta cualitativa a través de cuatro grupos de discusión ${ }^{8}$ y dos entrevistas ${ }^{9}$ (en formato online debido al contexto de confinamiento) que aportaron información útil para enriquecer el diagnóstico del sector. Un total de 27 organizaciones y redes del TSSE trasladaron de primera mano el impacto que estaba teniendo en las entidades y en las personas destinatarias la crisis de la covid $-19^{10}$.

Los resultados que se presentan en este artículo forman parte de un estudio más amplio en el que se detallan más situaciones y problemas que tanto las organizaciones como sus personas destinatarias estaban afrontando en abril de 2020, así como más detalles sobre la incidencia específica que dichas situaciones y problemáticas puedan estar teniendo atendiendo a diferentes variables independientes sobre las organizaciones.

estiman en 3.938 las organizaciones que actualmente forman parte de él. Con todo, los resultados de esta parte del estudio cuentan, a un nivel de confianza del $95 \%$, con un margen de error de $+/-7,05 \%$.

7 Los datos han de ser tomados con cierta cautela, en especial cuando se realizan desagregaciones. En este sentido, a través de la motivación de la respuesta al cuestionario durante el trabajo de campo se ha tratado de hacer seguimiento de la distribución de la muestra, procurando tener una representación lo más cercana posible al universo en variables como el territorio histórico, ámbito de actuación y tamaño de la organización, pero no con ello garantizando una muestra controlada. Así, puede decirse que la muestra de este estudio mantiene en lo esencial la heterogeneidad perseguida si bien, para una correcta interpretación de los datos, hay que tener en cuenta que atendiendo al peso del conjunto de las organizaciones vascas:

- Según el ámbito, están algo sobrerrepresentadas las organizaciones de Empleo y Servicios Sociales.

- Según territorio histórico hay una leve infrarrepresentación de las organizaciones de Gipuzkoa y mayor presencia de las de Araba (pese a que hayan contestado más organizaciones de Gipuzkoa que de Araba).

- Según tamaño, están infrarrepresentadas las organizaciones pequeñas y medianas (aquellas con menos de 300.000 euros en volumen de ingresos).

- Según contingencia atendida, hay mayor peso de las que atienden a personas en situación o riesgo de exclusión.

${ }^{8}$ Concretamente, tres grupos de discusión con organizaciones que atienden a personas en situación de discapacidad, a infancia y familia y a personas mayores y un grupo de discusión con redes y organizaciones que tienen actividad en los tres territorios históricos, con la intención de reflexionar sobre los matices concretos de cada territorio.

9 Concretamente, dos entrevistas a informantes clave de organizaciones con conocimiento específico sobre personas migrantes y mujeres que sufren violencia machista.

${ }_{10}$ Tanto en los grupos de discusión como en las entrevistas, la muestra de organizaciones y personas participantes es una muestra no probabilística de tipo opinático (muestra razonada) con la que no se busca la representatividad estadística del conjunto, sino la selección de aquellas personas u organizaciones que por su mayor conocimiento o motivación en el problema a investigar sean consideradas las más idóneas o mejores informantes. Los criterios de selección y composición para esta parte del trabajo de campo cualitativo han sido contrastados con el Departamento de Empleo y Políticas Sociales de Gobierno Vasco y Sareen Sarea atendiendo a principios de priorización por posible nivel de impacto de la crisis de la covid-19 o de necesidad de ampliar la información en relación con algunos colectivos. 


\section{El impacto en las personas destinatarias de las organizaciones del TSSE}

La limitación de circulación de personas impuesta por el Gobierno de España para la gestión de la crisis sanitaria ocasionada por la covid-19, tras la declaración del estado de alarma el 14 de marzo de 2020 , tuvo consecuencias de gran calado en las personas destinatarias de las organizaciones del TSSE.

Un contexto de confinamiento, común para toda la sociedad, en el que la mayoría de las personas tuvieron que vivir semanas cambiando sus rutinas, y se vieron obligadas a permanecer en casa, a cesar su actividad laboral o a limitar las salidas y contactos con otras personas. Contexto que se agrava si se piensa en los impactos que puede generar en personas en mayor situación de vulnerabilidad o exclusión, como personas sin hogar, en riesgo de pobreza, personas con discapacidad, personas mayores, infancia vulnerable, mujeres que sufren violencia o personas con enfermedad mental, entre otros colectivos, que han tenido que enfrentar muy diversas problemáticas. En ellas, el aislamiento social, la pérdida de empleo, la falta de rutinas y referentes, las limitaciones de los espacios de ocio o el confinamiento en hogares no seguros impactan de manera más grave por la situación de vulnerabilidad de la que parten.

De hecho, los problemas derivados del confinamiento (problemas psicológicos sobrevenidos o agravados, conflictividad, desatención, pérdida de referentes, etc.) junto a los asociados a la brecha digital (falta de conexión a Internet u ordenador, limitado manejo de estas herramientas, desinformación, retraso escolar, etc.), y a la falta de recursos económicos son las situaciones que mayor número de organizaciones identificaban en abril de 2020 en relación con sus personas destinatarias.

Gráfico 1. Problemas y situaciones que afrontaron en abril de 2020 y que preveían afrontar a corto plazo las organizaciones en relación con el impacto de la crisis de la covid-19 en las personas destinatarias (en porcentaje) psicológicos sobrevenidos o agravados, conflictividad, etc.)

Problemas derivados de la brecha digital (falta de conexión a Internet, ordenador, habilidades, etc.)

Falta de recursos económicos

Soledad no deseada (ausencia de red de apoyo)
agravada en esta situación

Problemas de atención sanitaria o deterioro de la salud

Aumento de la pobreza y/o privación material

Problemas educativos
(dificultad de seguir las clases, retraso escolar, etc.)

Problemas administrativos y jurídicos (detenciones, problemas con los permisos para transitar, etc.)

Problemas en el entorno comunitario (conflictividad, situaciones de riesgo, etc.)

Problemas de alimentación

\begin{tabular}{r} 
Problemas de alimentación \\
\hline Violencia, incluyendo violencia de género, \\
agravada por el confinamiento
\end{tabular}

Falta de atención en el domicilio (personas menores de edad, personas en situación de dependencia, etc.

Cambios en los perfiles de las personas destinatarias Empleo relacionado con los cuidados

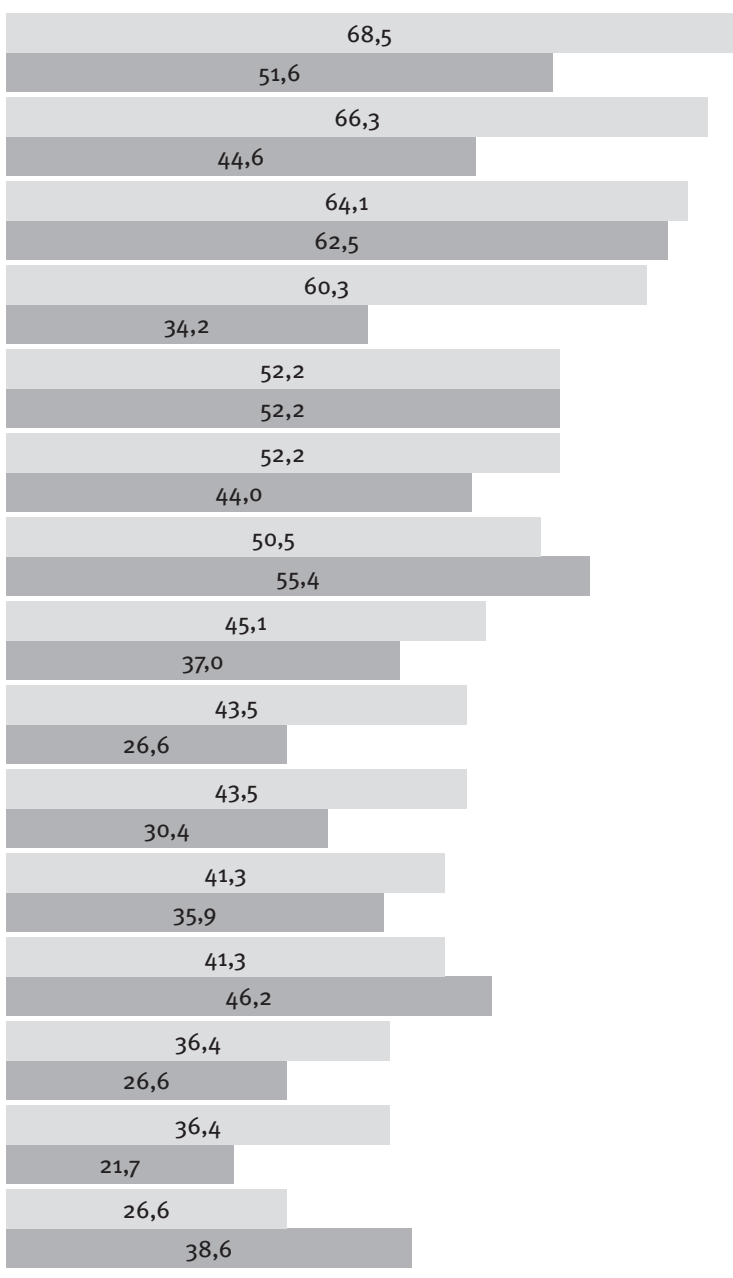

Abril 2020

Estimación 2020

Fuente: Elaboración propia. 
El 68,5\% de organizaciones afirmaba en abril de 2020 que sus personas destinatarias estaban experimentando problemas derivados del confinamiento, como problemas psicológicos sobrevenidos o agravados, conflictividad, desatención, pérdida de referentes, aumento de situaciones de violencia, aumento de situaciones de consumo, desánimo, etc. Estos problemas eran destacados en mayor medida por organizaciones que atienden a personas en situación o riesgo de dependencia y a personas con discapacidad. Además, más de la mitad de las organizaciones preveían entonces que, a corto plazo, sus personas destinatarias seguirían experimentando esos problemas relacionados con el confinamiento.

En torno a dos de cada tres organizaciones $(66,3 \%)$ percibía en abril de 2020 que sus personas destinatarias estaban experimentando problemas derivados de la brecha digital, por no disponer de ordenador o conexión a Internet, o por no tener habilidades relacionadas con la alfabetización digital. Esos problemas se estaban traduciendo en desinformación, dificultades para la comunicación online (videollamadas, etc.) o dificultades de acceso a formación online, entre otros. De cara a los siguientes meses, el 44,6\% de organizaciones apuntaba que sus personas destinatarias padecerían dichos problemas relacionados con la brecha digital. Aunque las organizaciones que atienden a personas en situación o riesgo de dependencia o desprotección señalaban en mayor medida la incidencia de los problemas derivados de la brecha digital en sus personas destinatarias, son numerosos los colectivos de personas destinatarias que experimentaban estos problemas derivados de la brecha digital: personas con discapacidad física e intelectual, personas mayores, personas en situación o riesgo de pobreza, personas migrantes, etc. En algunos casos se trataba de una cuestión de alfabetización digital (falta de conocimiento o habilidades para manejarse en entornos digitales) y en otros se trataba de falta de acceso a dispositivos tecnológicos o de conexión a Internet, principalmente por falta o escasez de recursos económicos.

La soledad no deseada o la falta de una red de apoyo agravada por la situación de confinamiento es una situación que el 60,3\% de las organizaciones percibía en relación con sus personas destinatarias. Esta cuestión era destacada en mayor proporción por organizaciones que atienden a personas en situación o riesgo de dependencia. De cara a los siguientes meses, la proporción de organizaciones que señalaba esta cuestión descendía notablemente, aunque el 34,2 \% de las organizaciones sostenía que esa soledad no deseada seguiría estando presente entre sus personas destinatarias.

El $64,1 \%$ de las organizaciones indicaba en abril de 2020 que sus personas destinatarias se estaban enfrentando a la falta de recursos económicos y, de cara a los siguientes meses, un porcentaje similar preveía que seguirían en esa misma situación. Se identificaban situaciones diversas relacionadas con la pérdida de empleo, con familias que antes de la crisis ya se encontraban en situación de precariedad y pobreza que habían visto agravada su situación, o con la falta de recursos para hacer frente al pago de medicinas. De hecho, son las organizaciones que atienden a personas en situación o riesgo de exclusión las que en mayor medida identificaban la falta de recursos económicos entre sus personas destinatarias, así como las que atienden a personas en situación o riesgo de desprotección.

El 52,2\% de las organizaciones indicaba que en ese momento sus personas destinatarias estaban afrontando la pérdida del trabajo remunerado. Se ve más señalada esta situación por organizaciones que atienden a personas en situación o riesgo de exclusión y desprotección; lo llamativo de la estimación para los siguientes meses es que el mismo porcentaje de organizaciones sostenía que en el corto plazo sus personas destinatarias seguirían afrontando dicha pérdida de trabajo remunerado.

El $50,5 \%$ de las organizaciones indicaba que sus personas destinatarias estaban afrontando un aumento de la pobreza y/o privación material. Este es un problema, señalado en más proporción por organizaciones que atienden a personas en situación o riesgo de exclusión, que lejos de mejorar, parece que empeora en la previsión que las organizaciones hacían para los meses siguiente, ya que el porcentaje de organizaciones que preveían que sus personas destinatarias afrontarían aumento de pobreza y/o privación material sube un $5 \%$.

Otras cuestiones que una amplia proporción de organizaciones identificaba en sus personas destinatarias eran problemas de atención sanitaria, educativos, administrativos y jurídicos, en entorno comunitario, de alimentación y de alojamiento. En concreto, el 52,2\% de las organizaciones apuntaba que sus personas destinatarias estaban en abril de 2020 padeciendo problemas de atención sanitaria o deterioro de la salud. El 45,1\% de organizaciones indicaba que sus personas destinatarias estaban afrontando problemas educativos tales como retraso escolar, dificultades para seguir las clases, pérdida de itinerarios educativos, etc. Esta cuestión era especialmente destacada por organizaciones que atienden a personas en situación o riesgo de desprotección. El 43,5\% de organizaciones sostenía que durante esas semanas del estado de alarma, sus personas destinatarias están enfrentando problemas administrativos y jurídicos, como detenciones o problemas con los permisos para transitar cuando es justificado. También un 43,5\% de organizaciones indicaba que sus personas destinatarias estaban experimentando problemas en el entorno comunitario, como problemas de conflictividad o situaciones de riesgo. El 41,3\% de organizaciones señalaba que sus personas destinatarias estaban afrontando problemas de alimentación y el 35,9\% indicaba que, en el corto plazo, sus personas destinatarias seguirán teniendo ese problema. 
También un 41,3\% de organizaciones indicaba que sus personas destinatarias estaban experimentando actualmente problemas de alojamiento, vivienda o exclusión residencial.

Por otro lado, el $26,6 \%$ de organizaciones ha registrado cambios en los perfiles de sus personas destinatarias y, de cara a los siguientes meses; aún más organizaciones preveían cambios en los perfiles, como indicaba el $38,6 \%$ de organizaciones. Esta diversificación de perfiles parece derivarse, entre otros factores, del aumento de personas atendidas. Por ejemplo, organizaciones de salud mental destacaban que había habido un aumento del número de personas que se dirigen a la organización que no tenían ninguna vinculación previa con ella, además de las personas que son habitualmente atendidas.

Además, un $20,7 \%$ de las organizaciones ha indicado que sus personas destinatarias están afrontando otro tipo de problemas que apuntan principalmente a: falta de apoyos profesionales $\mathrm{y} / \mathrm{o}$ terapéuticos en la vida diaria; reorganizaciones familiares para poder atender a personas en situación de dependencia; ralentización de actividades vitales para la mejora de la calidad de vida de las personas; toma de medidas especiales de confinamiento para determinados perfiles de personas (en alta exclusión, sin hogar, con enfermedad mental, etc.); procesos psicoterapéuticos y psicoeducativos suspendidos; dificultades de ofrecer un recogimiento emocional adecuado al no darse atención directa; impactos aún no previstos en personas destinatarias en países del sur; imposibilidad de asistir a grupos de ayuda mutua; e, incluso, ruptura con las dinámicas de interacción grupales generando en algunos casos gran impacto en la salud mental y emocional de las personas destinatarias.

\section{El impacto en las organizaciones del TSSE}

El impacto que las primeras semanas de la crisis de la covid-19 tuvo en las organizaciones del TSSE afectó tanto a la actividad externa que realizan como a la vida interna de las propias organizaciones. En la primera fase del estado de alarma, las organizaciones del TSSE vivieron momentos de mucha complejidad por el obligado cierre o reorientación de actividades, por el aumento de la atención directa en algunos servicios o por el impacto económico en la financiación de su actividad.

\subsection{Servicios y actividades}

Con el advenimiento de la crisis de la covid-19, numerosos servicios y actividades que prestan y desarrollan las organizaciones del TSSE enfocadas a las personas destinatarias se vieron afectadas. La modificación o reorientación de servicios o actividades y la suspensión o cancelación de actividades concretas fueron en abril de 2020 los problemas más extendidos entre las organizaciones.

En concreto, un $83,2 \%$ de organizaciones indicaban que estaban enfrentado una modificación o reorientación de servicios o actividades, por ejemplo, convirtiendo actividades a formato online o realizando acompañamiento telefónico. De cara a los meses siguientes, un $48,9 \%$ de ellas preveía que esa modificación o reorientación de servicios o actividades se mantendría.

En abril de 2020 , un $81 \%$ de organizaciones había visto alguna de sus actividades canceladas o suspendidas, como actividades presenciales formativas y de integración social, excursiones para personas con discapacidad, campamentos para infancia, refuerzo escolar en infancia vulnerable, clases de castellano necesarias para la integración social de personas migradas, talleres grupales en pisos con personas con enfermedad mental, etc. En la previsión para los siguientes meses, era el 41,8\% el porcentaje de las organizaciones que preveían tener actividades suspendidas.

Un 48,9\% de organizaciones había tenido que reducir la atención a personas destinatarias (disminución de consultas, cese de intervenciones, etc.) y un $22,8 \%$ preveía que esa reducción de atención se iba a mantener en los próximos meses. Por otro lado, el $33,2 \%$ de organizaciones estaba en abril de 2020 enfrentando una sobrecarga en la atención directa (apoyo a personas sin hogar, residencias de personas mayores o personas con discapacidad, servicios residenciales para mujeres que sufren violencia, etc.). Ese porcentaje subía en la estimación para los siguientes meses de 2020 , ya que un $44,6 \%$ de organizaciones preveía que tendría situaciones de sobrecarga en la atención en el corto plazo.

Un 46,2\% de organizaciones había tenido que cerrar algún servicio (centros de día, centros de ocupación, cierre de servicios de ocio, de respiro, servicios de venta al público, ludotecas, empresas de inserción, etc.). En concreto, entre quienes habían visto cerrar alguno de sus servicios, casi tres de cada diez consideraba que dichos servicios corrían el riesgo de no poder volver a abrirse por cuestiones relacionadas con la incertidumbre en los recursos económicos (principalmente de la financiación pública) o las condiciones de distanciamiento social. Se trataba principalmente servicios de hostelería, catering, venta y limpieza, actividades grupales (acciones de encuentro, etc.), actividades de respiro y centros de día o talleres o actividades vinculadas con infancia y juventud: actividades de ocio y gestión de recursos como ludotecas o colonias de verano, servicios vinculados a los centros educativos (apoyo, etc.), programas de atención a adolescentes en acogimiento residencial, proyectos dirigidos a jóvenes inscritos en el sistema de garantía juvenil, etc.

Por otro lado, el $38 \%$ de organizaciones había incorporado nuevos servicios o actividades como 
Modificación o reorientación de servicios o actividades (conversión a formato online)

Suspensión o cancelación de actividades concretas

Falta de material sanitario

Reducción de atención a las personas destinatarias

Cambios en las demandas de las personas destinatarias de los servicios

Cierre de servicios

Aumento de actividad de otras funciones sociales (sensibilización, denuncia, promoción, etc.)

Incorporación de nuevos servicios o actividades

Falta de información sobre la situación de las personas destinatarias (confinadas)
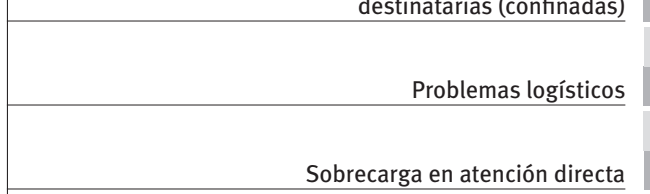

Problemas administrativos y/o jurídicos

Problemas de seguridad

Falta de recursos humanos

Abril 2020

Estimación 2020

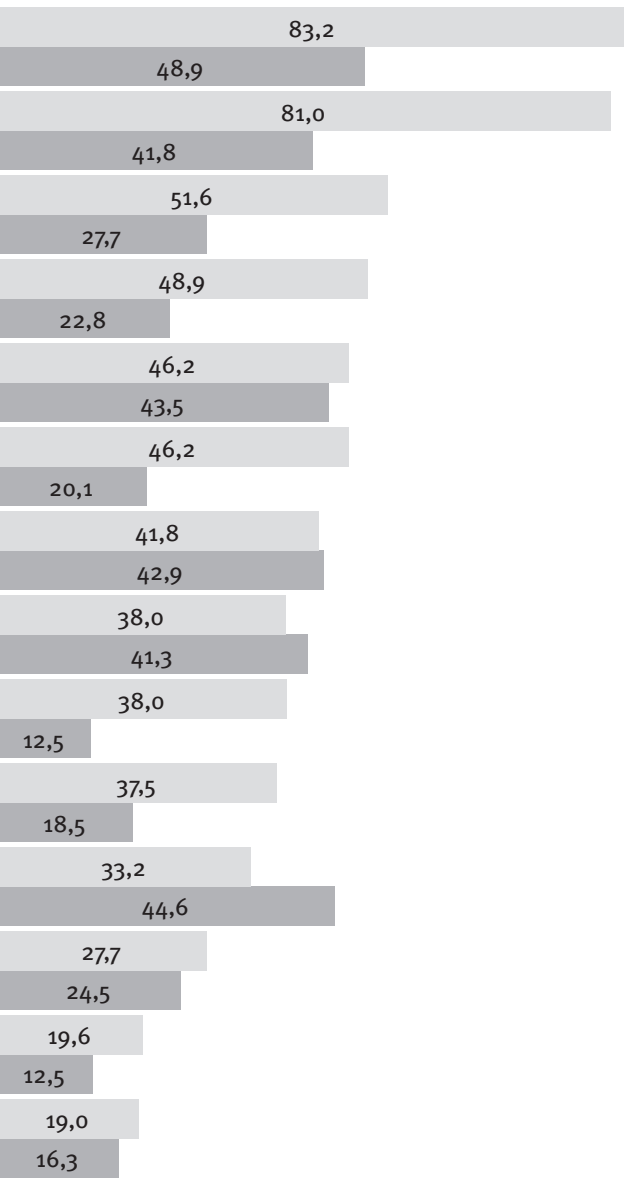

Fuente: Elaboración propia.

la apertura de servicios para atender situaciones urgentes, servicios en respuesta a las nuevas necesidades derivadas de la emergencia o servicios de atención a perfiles de personas destinatarias hasta ahora ocultos. La incorporación de nuevos servicios y actividades se produjo en mayor medida en organizaciones orientadas a personas en situación o riesgo de dependencia y en organizaciones grandes.

El $51,6 \%$ de organizaciones indicaba que enfrentaba problemas por la falta de material sanitario en los servicios, especialmente de atención directa (escasez de EPI, falta de espacios de aislamiento, test para actividades no sujetas al estado de alarma, etc.). El dato en la previsión para los siguientes meses descendía hasta el $\mathbf{2 7 , 7} \%$ de organizaciones. Los problemas de falta de material sanitario se produjeron sobre todo entre organizaciones que atienden a personas en situación o riesgo de dependencia 0 a personas con discapacidad y en organizaciones grandes.
El 46,2\% de organizaciones indicaba cambios en las demandas de las personas destinatarias a los servicios, tales como ayuda para realizar trámites online, ayudas económicas para cubrir necesidades básicas (alimentación, alquiler, etc.), mayor información sanitaria sobre la covid-19 y sobre el acceso a recursos, apoyo telefónico (psicológico, para regular la relación con hijos e hijas, etc.), demandas de atención y escucha y de comunicación online, petición de actividades online para evitar la soledad o demandas de apoyo educativo.

El 41,8\% de organizaciones había aumentado en abril de 2020 su actividad vinculada a la incidencia, la sensibilización, la denuncia o la promoción de derechos. Ese porcentaje sube ligeramente en relación con la previsión para los siguientes meses, ya que el $42,9 \%$ de organizaciones indicaba que preveía aumentar dicha actividad.

Las organizaciones también tuvieron que enfrentar, aunque en menor proporción, otros problemas 
como falta de información sobre la situación de las personas destinatarias confinadas, problemas logísticos, problemas administrativos o jurídicos, problemas de seguridad o falta de recursos humanos. Asimismo, algunas organizaciones destacaban dificultades relacionadas con las gestiones de las ayudas para mantener actividades y servicios, la coordinación con las Administraciones Públicas (en ocasiones satisfactoria y otras veces dificultosa) y los cambios continuos de protocolo durante aquellas semanas del estado de alarma.

\subsection{Personas y recursos económicos de las organizaciones}

La situación de las personas voluntarias y las personas remuneradas que forman los equipos de trabajo de las organizaciones del TSSE se vio afectada desde el comienzo de la crisis de la covid-19 de diversas maneras, y las organizaciones tuvieron que afrontar diversos problemas en relación con la gestión de los equipos de trabajo. Los más extendidos fueron las dificultades de gestión de la actividad por el cambio sustancial en las condicionales laborales (teletrabajo, conciliación, etc.), los riesgos psicosociales (situaciones de estrés, desgaste emocional, etc.) y los cambios en las condiciones de contratación.

En concreto, en abril de 2020 un $69 \%$ de organizaciones apuntaba que estaba teniendo dificultades de gestión de la actividad por el cambio sustancial en las condiciones laborales. Se trataba de situaciones vinculadas con la conciliación, la modificación de horarios de parte de la plantilla, el cambio al teletrabajo, la falta de competencias digitales que dificultaban la comunicación o la falta de práctica en teletrabajo, la falta de equipos y otras herramientas telemáticas, etc. Esta es una cuestión que destaca especialmente entre organizaciones que atienden a personas en situación o riesgo de desprotección.

Un 63,6\% de organizaciones apuntaba que sus personas voluntarias y remuneradas estaban enfrentando riesgos psicosociales (estrés, desgaste emocional, ansiedad, gran dedicación, etc.). Esta cuestión destacaba en mayor medida en organizaciones que atienden a personas en situación o riesgo de dependencia y en organizaciones grandes. Sobre todo, destacaba la sobrecarga que esta situación estaba provocando en los equipos de atención directa, sumada a la tensión del confinamiento o equipos de intervención que no contaban con suficientes herramientas para gestionar una situación de tal envergadura. Es el caso de personas profesionales que tuvieron que atender a personas afectadas por la covid-19, realizar atención de llamadas telefónicas de personas usuarias para el desahogo, hacer seguimiento de menores en familias desestructuradas en situación de confinamiento, o profesionales bajo situación de estrés en los equipos de los centros residenciales. En la previsión a corto plazo, un $48,4 \%$ de organizaciones preveía que sus personas voluntarias y remuneradas enfrentarían riesgos psicosociales.

Un 60,9\% de organizaciones estaba afrontando cambios en las condiciones de contratación. Se trataba de cambios relacionados con la imposibilidad de garantizar la continuidad de algunos contratos,

Gráfico 3. Problemas y situaciones que afrontaron en abril de 2020 y que preveían afrontar a corto plazo (2020) las organizaciones en relación con el impacto de la crisis del covid-19 en las personas voluntarias y remuneradas (\%)

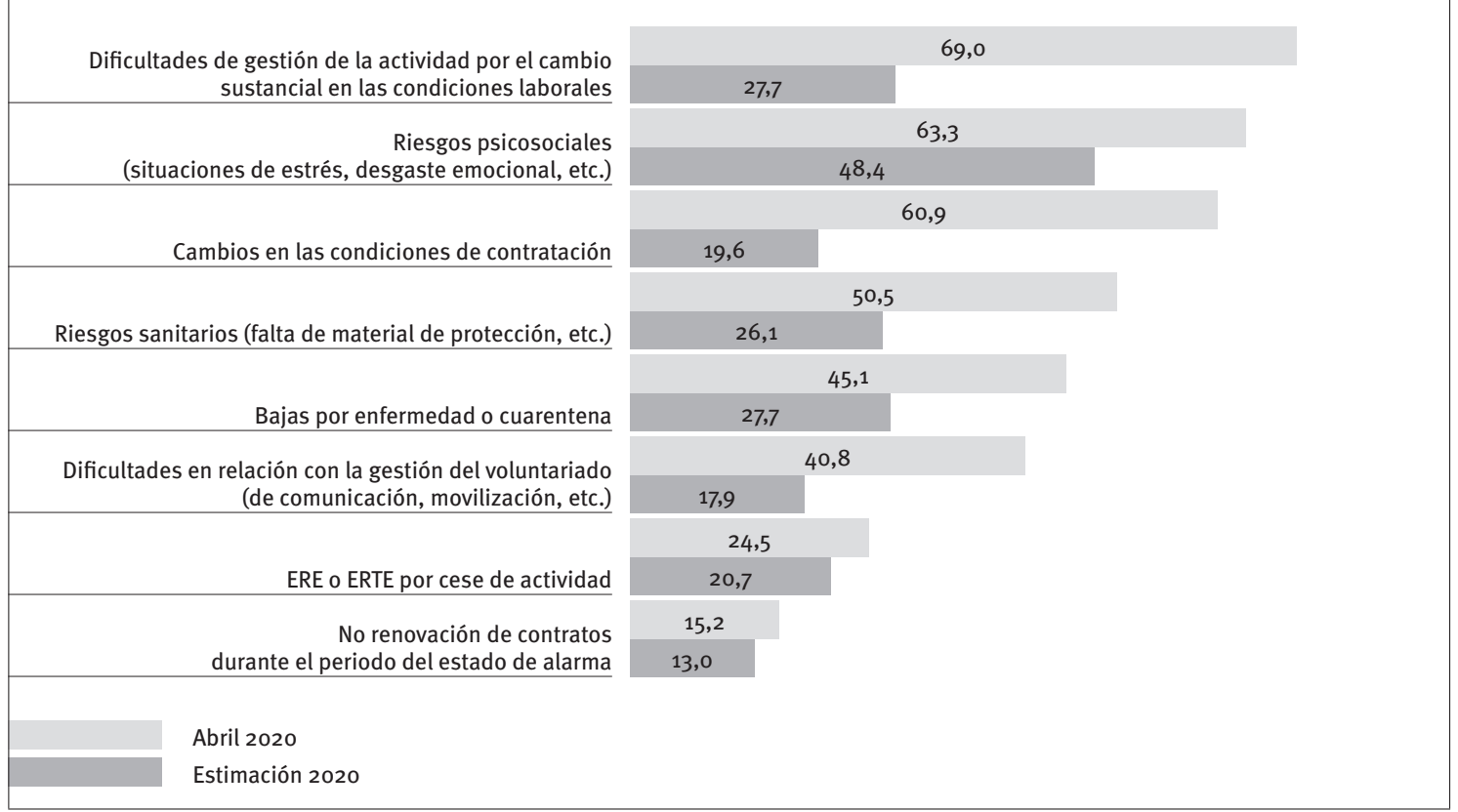

Fuente: Elaboración propia. 
cambios en las tareas, contratos que no iban a poder ser renovados, disminución de horas en los contratos, personas autónomas con las que trabajaban las entidades y que no podían facturar ni acceder a ayudas, suspensión de las personas en prácticas, etc. Estos cambios en las condiciones de contratación afectaron en mayor proporción a organizaciones que atienden a personas en situación o riesgo de dependencia y a personas con discapacidad.

En relación con dichos cambios en las condiciones de contratación, concretamente, el 47,8\% de organizaciones había tenido que realizar cambios 0 ajustes en las tareas y un $41,8 \%$ cambios en la jornada laboral por ajustes de la actividad.

Asimismo, un $26,1 \%$ de organizaciones había enfrentado cambios en la jornada laboral de sus personas remuneradas para atender a familiares $y$ un $7,6 \%$ indicaba tener excedencias para atender a familiares. Un 8,1\% de organizaciones indicaba tener que estar realizando horas extra y un $6 \%$ había enfrentado reducción de salarios.

En torno a la mitad de las organizaciones (50,5\%) indicaba estar afrontando en abril de 2020 riesgos sanitarios vinculados al personal (falta de material de protección, test, etc.). Además, un 45,1\% de organizaciones identifica bajas por enfermedad 0 cuarentena entre sus personas remuneradas.

Un 40,8\% de organizaciones estaba afrontando actualmente dificultades en la gestión del voluntariado, como dificultades de comunicación, movilización, falta de criterios claros de actuación, necesidad de ofrecer formación adaptada al nuevo marco, incremento del voluntariado online, adaptación de horarios y tareas, cancelación de la actividad voluntaria, perdida de relación con el voluntariado, cese de actividad de voluntariado de más edad por riesgo al contagio, etc. De cara a los siguientes meses, un $17,9 \%$ de organizaciones estimaba afrontar dichas dificultades en relación a la gestión del voluntariado. No obstante, frente a la adversidad las entidades también han visto un mayor compromiso de las personas voluntarias, una implicación mayor si cabe para realizar las tareas que se precisaran.

En menor proporción, las organizaciones del TSSE también enfrentaron ERE o ERTE (24,5\%) y la no renovación de contratos durante el estado de alarma (15,2\%). En ambos casos, la previsión para los siguientes meses apuntaba a unos porcentajes ligeramente menores.

Gráfico 4. Distribución de organizaciones según existencia de cambios en las condiciones de contratación (en porcentaje)

\begin{tabular}{|c|c|c|}
\hline Cambios o ajustes en las tareas & 47,83 & 52,17 \\
\hline Cambios en jornada por ajustes de actividad & 41,85 & 58,15 \\
\hline \multirow[t]{2}{*}{ Cambios en jornada para atender familiares } & 26,09 & 73,91 \\
\hline & 8,15 & 91,85 \\
\hline Excedencias para atender a familiares & 7,61 & 92,39 \\
\hline Reducción de salarios & 5,98 & $94,, 02$ \\
\hline \multicolumn{3}{|l|}{ Sí } \\
\hline \multicolumn{3}{|l|}{ No } \\
\hline
\end{tabular}

Fuente: Elaboración propia. 


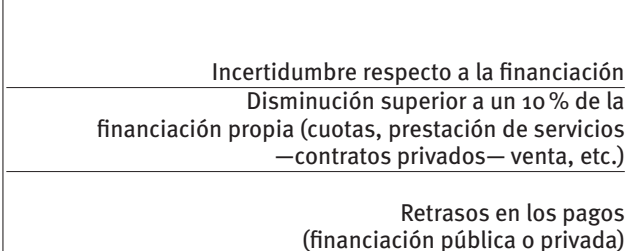

Suspensión de contratos o subvenciones públicas

Falta de liquidez/necesidad de préstamos

Dificultades de un número importante de personas para mantener cuotas y/o copagos

Aumento de la financiación pública para responder al impacto de la crisis sanitaria

Aumento superior a un $10 \%$ de la financiación pública

Aumento superior a un $10 \%$ de donaciones y legados

Abril 2020

Estimación 2020

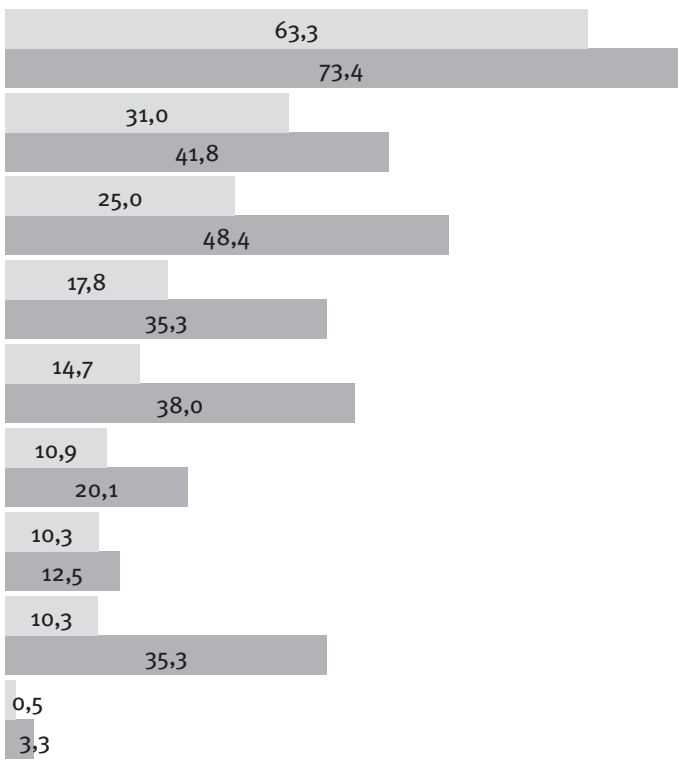

63,3

4,7

0,9

20,1

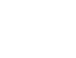

Fuente: Elaboración propia.

La situación económica de las organizaciones se ha visto fuertemente afectada por la situación generada a partir del estado de alarma. Además de problemas concretos que ya estaban afrontando, la incertidumbre sobre cómo se vería afectada la financiación era grande. De hecho, la incertidumbre respecto a la financiación era en abril de 2020 y en la previsión a corto plazo el problema más extendido entre las organizaciones. También son destacables la disminución superior a un $10 \%$ de la financiación propia en abril de 2020 y la previsión de retrasos en los pagos públicos o privados. A diferencia de lo que ocurre con los problemas o situaciones vinculadas a las personas destinatarias, los servicios o el personal remunerado, donde parece que la previsión en los próximos meses es de mejora, la previsión sobre todos los problemas relativos a los recursos económicos es que se vean agravados a lo largo de 2020.

En concreto, un 63,6\% de organizaciones indicaba estar viviendo incertidumbre respecto a la financiación y un 73,4 \% estimaba que la viviría a corto plazo. Aunque es una tendencia generalizada ente las organizaciones, esta preocupación es algo superior en organizaciones que atienden a personas en situación o riesgo de dependencia y a personas con discapacidad. Esta era, sin duda, una de las cuestiones que más preocupaba a las organizaciones ya que pone en tela de juicio su supervivencia. Eran generalizadas las dudas sobre cuándo se resolverían las ayudas y subvenciones y si habría una merma en las cuantías de subvenciones o convenios futuros. Se ponía también sobre la mesa el incremento de la carga de trabajo pese a haberse suspendido actividades, el acceso a líneas de financiación vinculadas a la emergencia sanitaria, las repercusiones de la pérdida producida en la financiación privada, el posible reembolso de los gastos generados por la emergencia social, la imposibilidad de hacer frente a subidas salariales ya pactadas o a gastos estructurales (alquiler, suministros, seguros, etc.).

En relación con la financiación propia (cuotas, prestación de servicios, eventos, venta de productos, campañas, etc.), un $31 \%$ de organizaciones estaba en abril de 2020 afrontando una disminución superior a un $10 \%$ en ella. En la estimación para los siguientes meses, un $41,8 \%$ de organizaciones indicaba que preveía dicha disminución en su financiación propia.

El $25 \%$ de organizaciones estaba afrontando en aquellas semanas retrasos en los pagos, de origen público o privado y, a corto plazo, un $48,4 \%$ de organizaciones preveía enfrentarlos. Dichos retrasos afectaban en mayor proporción a organizaciones que atienden a personas en situación o riesgo de exclusión y a organizaciones grandes. Además, un $17,8 \%$ de organizaciones estaba enfrentando suspensión de contratos o subvenciones públicas, lo cual ponía en riesgo la continuidad de algunos proyectos e implicaba despidos; en la previsión a corto plazo, el porcentaje se elevaba a un 35,3\%.

Un $14,7 \%$ de organizaciones estaba en abril de 2020 teniendo problemas de liquidez o necesidad de préstamos. De cara a los próximos meses, un $38 \%$ 
de organizaciones preveía tener falta de liquidez 0 necesidad de préstamos. Este contexto genera gran incertidumbre con respecto a la imposibilidad de pagar salarios y de ofrecer servicios nucleares.

Casi el $11 \%$ de organizaciones indicaba dificultades de un gran número de personas para mantener cuotas y/o copagos (reducción de cuotas de personas socias, impago de copagos, etc.). En la previsión a corto plazo, un $20,1 \%$ de organizaciones estimaba que tendría dichas dificultades.

Por otro lado, el 10,3\% de organizaciones había visto aumentar su financiación pública para responder al impacto de la crisis sanitaria y un $12,5 \%$ preveía que su financiación pública aumentará por dicho motivo. También en torno al $10 \%$ estaba afrontando una disminución superior al $10 \%$ de su financiación pública. Para 2020, el 35,3\% de organizaciones así lo preveía.

Además, un $13 \%$ de organizaciones indicaba estar afrontando otros problemas en relación con los recursos económicos, que sobre todo tenía que ver con bajadas en los pedidos de venta de productos, ajustes futuros a las subidas salariales previstas en convenio, servicios que habían dejado de ofrecerse pero que generaban un gasto incluso estando inactivos, requisitos exhaustivos de las administraciones públicas para justificar la actividad que se estaba realizando, adelanto de gasto o gasto imprevisto no presupuestado.

\section{Algunas reflexiones finales}

Los datos del estudio ofrecen una fotografía de la situación que las organizaciones del TSSE y sus personas destinatarias estaban viviendo durante la primera etapa del confinamiento (abril 2020), unas semanas de gran complejidad en la gestión y articulación de los servicios y actividades de las organizaciones y de grandes dificultades para muchas personas destinatarias. Esa fotografía permite entender las primeras respuestas de las organizaciones ante la crisis y la realidad inicial que el confinamiento supuso para las personas destinatarias. Evidentemente, algunas de las problemáticas han cambiado según ha ido avanzando la desescalada y la previsión de las propias organizaciones apuntaba a que el peso de las distintas problemáticas iba a ir variando con el paso de las semanas, pero la significatividad del impacto inicial permite comprender el alcance que la crisis pueda tener tanto en el TSSE como en las personas destinatarias.

A diferencia de lo que ocurre con los problemas o situaciones vinculadas con las personas destinatarias, los servicios o el personal remunerado, donde parece que la previsión los siguientes meses de 2020 mejora, la previsión sobre todos los problemas relativos a los recursos económicos es que se vean agravados a lo largo de 2020.
Las organizaciones ya indicaban que parte de los problemas y situaciones que estaban afrontando en abril de 2020 sus personas destinatarias iban posiblemente a minimizarse una vez superada la fase crítica de esta crisis social. Sin embargo, de cara a los siguientes meses, las organizaciones apuntaban que la falta de recursos económicos, el aumento de la pobreza y/o privación material y la pérdida de trabajo remunerado iban a ser los problemas más extendidos entre sus personas destinatarias (más de lo que ya lo estaban siendo), lo cual resulta alarmante si tenemos en cuenta que los perfiles de personas atendidas por muchas de las entidades sociales se caracterizan ya por ser personas en riesgo o situación de exclusión. De cara a la labor de las organizaciones destaca también el cambio de perfiles en las personas atendidas que se preveía para los meses restantes de 2020.

Algunas cuestiones que preocupaban especialmente a las organizaciones en relación a las personas destinatarias tienen que ver con la invisibilización $y$, en algunos casos, la estigmatización que algunos colectivos han vivido, y con la importancia de las redes naturales de apoyo durante el confinamiento.

Durante las primeras semanas del estado de alarma, era llamativo que en el discurso público muchas de las personas destinatarias de las organizaciones del TSSE estaban siendo invisibilizadas. Salvo algunas excepciones, la opinión pública, los medios de comunicación o las redes sociales no estaban recogiendo la situación específica que personas con discapacidad, mujeres o infancia podían estar viviendo (al menos en los inicios). Esa invisibilidad preocupaba a las organizaciones porque no ponía ningún foco, social o mediático, sobre ellas.

Ante la emergencia sanitaria, la prioridad se focalizó en generar una respuesta institucional especialmente dirigida hacia algunos colectivos prioritarios. Sin embargo, con el transcurso de las semanas, no se fueron incorporando al foco social otros colectivos vulnerables cuya situación estaba siendo también especialmente grave. Si ya de por sí muchas personas destinatarias de las organizaciones del TSSE viven invisibilizadas socialmente, la crisis de la covid-19 no parece haberlas hecho más visibles.

En paralelo, algunos colectivos de personas destinatarias, que sí estaban recibiendo atención mediática, fueron señalados e incluso estigmatizados, como fue el caso de personas migrantes o personas mayores. En relación con las primeras, el impacto mediático que estaban teniendo algunas actuaciones concretas o los bulos que circulaban en torno a la inmigración generaron una afectación a nivel de imagen y estigmatización. En relación con las personas mayores, su estigmatización como colectivo de riesgo generó una afectación en su estado anímico y en su rol social. El miedo y la preocupación que vivieron durante las semanas de confinamiento siguen actualmente siendo palpables, sobre todo en lo relacionado con 
su esfera social y relacional, con el espacio social y simbólico que les va a dejar la sociedad cuando la crisis de la covid-19 se supere.

Por otro lado, el papel de las familias y las redes naturales de apoyo de las personas destinatarias fue fundamental durante el confinamiento, de gran valor para las propias destinatarias y para las organizaciones del TSSE. Especialmente en el caso de algunos colectivos de personas destinatarias se erigieron en pilar fundamental, reorganizándose para ver cómo podían dar mejores apoyos dentro del domicilio, modificando incluso la convivencia. Fue, por ejemplo, el caso de personas con problemas de salud mental que vivían solas y que se trasladaron a vivir con sus padres antes de que se decretase el estado de alarma. 0 el de las personas con discapacidad intelectual, cuyas familias mantuvieron estoicamente la convivencia sin momentos de respiro. El valor de esos cuidados proporcionados por las familias fue especialmente importante en el caso de personas muy dependientes. Asimismo, en términos generales, las familias con niños y niñas también asumieron un papel fundamental, apoyando a sus hijas e hijas a nivel educativo, pero también a nivel emocional durante el confinamiento.

En la estimación a corto plazo, un amplio porcentaje de organizaciones preveía que se mantendría la modificación o reorientación de servicios o actividades como problema y que se incrementaría la sobrecarga en la atención directa, la incorporación de nuevos servicios o actividad y el aumento de la actividad vinculada a otras funciones sociales (detección de necesidades, acogida, sensibilización y denuncia, etc.). Con vistas al futuro, las organizaciones preveían distintas situaciones en relación con sus servicios y con la actividad que realizan: reestructuración de actividad, replanificación de servicios y tareas, ajuste de servicios a los presupuestos, posible cobro de servicios que venían siendo gratuitos, imposibilidad de realizar las horas de servicio pactadas o los criterios establecidos con los organismos públicos financiadores, dificultades de algunas personas destinatarias para reincorporarse a los servicios, refuerzo de programas psicoterapéuticos y psicoeducativos que se han visto interrumpidos y que han mermado la intervención con adolescentes con problemas de conducta, incremento del número de plazas para la atención a situaciones de exclusión, adaptación de actividades a nuevas respuestas, mayor coordinación entre el ámbito sanitario y el social, incertidumbre ante los protocolos de seguridad una vez superada la etapa actual, dificultades de activar actividades de tiempo libre socioeducativo (campamentos, colonias, etc.).

En relación con las personas voluntarias y remuneradas, más allá de las dificultades de gestión que se estaban encontrando las organizaciones por los cambios en las condiciones laborales o de contratación, la estimación para el corto plazo apuntaba a que los riesgos psicosociales son el problema que mayor porcentaje de organizaciones preveía que sus personas voluntarias y remuneradas tendrían que afrontar.

Es destacable que si bien el alcance de muchas de las situaciones que las personas destinatarias y los servicios y actividades de las organizaciones han vivido en los inicios del confinamiento ha sido especialmente grave, la previsión para los próximos meses que las organizaciones realizan pone el peso en los recursos económicos. Cualquiera de las problemáticas analizadas se observa más generalizada en la previsión para el resto del 2020 que en abril de 2020. La incertidumbre en la financiación y los retrasos en los pagos se apuntan como los problemas más acuciantes para los siguientes meses.

Algunas cuestiones que preocupaban especialmente a las organizaciones en relación a sus servicios y recursos tienen que ver con la necesaria adaptación de los servicios, así como la incertidumbre sobre los mismos y sobre los recursos económicos. El cierre de servicios durante el estado de alarma, como servicios de atención diurna o centros de días y servicios de tiempo libre para infancia o personas con discapacidad, ya preocupaba en abril de 2020 por el impacto en la sostenibilidad de las organizaciones.

A pesar de la incertidumbre y la urgencia vivida durante las primeras semanas del estado de alarma, las organizaciones del TSSE buscaron estrategias para adaptarse al contexto y seguir ofreciendo respuestas a sus personas destinatarias. Esa adaptación va más allá de la modificación de la manera en que se ofrecen servicios y actividades. La reconversión hacia el teletrabajo en los servicios de apoyo por ejemplo ha sido una innovación forzada que quizás en algunos casos se pueda incluso mantener pasado el confinamiento por los buenos resultados que está dando. Se están, en este sentido, descubriendo formas distintas, forzadas por el contexto, de ofrecer servicios y actividades.

Las organizaciones manifestaban en abril de $\mathbf{2 0 2 0}$ una preocupante incertidumbre principalmente vinculada con la financiación de las organizaciones: existía una gran preocupación por la viabilidad de actividades y servicios. La incertidumbre sobre lo que iba a pasar con la financiación pública se focaliza en el temor a que las mayores partidas económicas vayan destinadas al ámbito sanitario y no haya una apuesta específica por lo social. A ello se une la carga económica que para muchas organizaciones estaba suponiendo hacer frente al contexto de estado de alarma con recursos propios. Con todo, el contexto dibujaba una situación poco sostenible en el tiempo.

Ante esta incertidumbre, algunas organizaciones temen por su supervivencia, especialmente las pequeñas y medianas y las que cuentan con mayoría de personas voluntarias en sus equipos de trabajo, cuya financiación depende principalmente de subvenciones públicas. 
Parece importante seguir analizando el impacto que van a vivir las organizaciones sociales y sus personas destinatarias a corto y medio plazo, y es que las previsiones no apuntan al optimismo. En este sentido visibilizar y seguir apoyando la insustituible labor que realizan estas entidades resulta clave, ya que representan el sostén de colectivos que se enfrentan a múltiples situaciones de vulnerabilidad que se magnifican en contextos de crisis como el actual. 


\section{Referencias bibliográficas}

GOBIERNO VASCO (2016): Ley 6/2016, de 12 de mayo, del Tercer Sector Social de Euskadi, Boletín Oficial del País Vasco, no 94, 19 de mayo, 〈http://www. euskadi.eus/bopv2/datos/2016/05/1602096a. pdf〉.
OBSERVATORIO VASCO DEL TERCER SECTOR SOCIAL (2020): El Tercer Sector Social de Euskadi ante la situación de estado de alarma provocada por el Covid19, Vitoria-Gasteiz, Gobierno Vasco, 〈https://3seuskadi.eus/wp-content/uploads/ Informe_COVID_19.pdf . 